\title{
Ruptura espontânea de artéria uterina por foco de endometriose: relato de caso
}

\author{
Spontaneous rupture of the uterine artery by focus of endometriosis: case report \\ Cecília de Carvalho Contin Poletto ${ }^{1}$, Lorena Anunziato Sant'Ana ${ }^{1}$, Maria Izabel Mizoguchi Guerra ${ }^{1}$, \\ Mariana Baraldi Mafra', Solena Ziemer Kusma ${ }^{3}$, Sheldon Rodrigo Botogoski ${ }^{4}$, \\ Talita Cristiane de Lima Guerra ${ }^{1}$
}

\section{Resumo}

Endometriose é uma afecção clínica ginecológica benigna, recorrente, estrogênio-dependente definida pela presença de glândulas e estroma endometriais fora da cavidade uterina. As manifestações clínicas principais são dor pélvica crônica e infertilidade. Este distúrbio possui difícil diagnóstico, sendo realizado definitivamente por análise patológica da biópsia da lesão por meio de laparoscopia ou laparotomia. Na gestação, a endometriose pode causar graves complicaçôes, a exemplo do hemoperitônio por ruptura de vasos útero-ovarianos. Este relato de caso de paciente com 27 anos, primigesta, idade gestacional ultrassonográfica de 36 semanas, com quadro de abdômen agudo, que evoluiu para choque hipovolêmico. Durante a exploração cirúrgica foi identificado como causa do hemoperitônio a ruptura da artéria uterina esquerda, onde foi observada a presença de fibrose e foco de endometriose. O material obtido no procedimento foi encaminhado para análise patológica, que confirmou a presença de tecido endometriótico no vaso uterino. Como este caso apresenta uma rara complicação ocasionada pela endometriose na gestação, é de suma importância relatá-lo para atentar médicos, em busca de diagnóstico e tratamento

1. Acadêmica da Escola de Medicina da Pontifícia Universidade Católica do Paraná - $5^{\circ}$ ano do curso de Graduação

2. Médica Residente de Ginecologia e Obstetrícia do Hospital Municipal e Maternidade Escola Dr. Mário de Moraes Altenfelder Silva - Vila Nova Cachoeirinha

3. Professora de Metodologia da Pesquisa e Epidemiologia Clinica da Escola de Medicina daPontifícia Universidade Católica do Paraná

4. Professor Adjunto da Escola de Medicina da Pontifícia Universidade Católica do Paraná e Professor Adjunto do Departamento de Tocoginecologia da Universidade Federal do Paraná

Trabalho realizado: Pontifícia Universidade Católica do Paraná. Departamento de Ginecologia e Obstetrícia.

Endereço para correspondência: Maria Izabel Mizoguchi Guerra. Rua Guilherme Pugsley, 1230, apto. 802 - Água Verde80620000 -Curitiba-PR - Brasil. E-mail:bel-guerra@gmail.com Conflito de interesses: Todos os autores declaram não haver nenhum potencial conflito de interesses referente a este artigo. precoces, garantindo um melhor prognóstico materno fetal.

Descritores: Endometriose, Complicações na gravidez, Hemoperitônio, Choque, Artéria uterina

\begin{abstract}
Endometriosis is a recurrent benign estrogen-dependent gynecological disease defined by the presence of endometrial glands and stroma located out of uterus. The main clinical manifestations are chronic pelvic pain and infertility. This disorder has difficult diagnosis, that is confirmed by pathological analysis of the lesion's byopsy through laparoscopy or laparotomy. During pregnancy, endometriosis can cause serious complications, such as hemoperitoneum by rupture of utero-ovarian vessels. This study aims to report the case of a 27-year-old, primigravida, ultrasound gestational age of 36 weeks, with acute abdomen that envolved to hypovolemic shock. During a surgical exploration, it was identified a rupture of the left uterine artery as the cause of hemoperitoneum, also the presence of fibrosis and focus of endometriosis. The material obtained was referred for biopsy, which confirmed the presence of uterine non-vessel endometrial tissue. This case presents a rare complication of endometriosis during pregnancy, which means a significant importance for the awareness of physicians in order to guarantee an early diagnosis and treatment, ensuring a better maternal and fetal prognosis.
\end{abstract}

Keywords: Endometriosis, Pregnancy complications, Hemoperitoneum, Shock, Uterine artery.

\section{Introdução}

Endometriose é uma afecção clínica ginecológica benigna recorrente definida pela presença de glândulas e estroma endometriais fora da cavidade uterina ${ }^{(1-2)}$. É uma patologia estrogênio dependente com fisiopatologia desconhecida. Diversas teorias propõem explicações para sua causa, entre elas: teoria da menstruação retrógrada na qual haveria uma aderência de 
tecido endometrial pós-menstrual na cavidade peritoneal e demais órgãos em decorrência de fluxo tubário retrógrado pelas trompas de Falópio em associação a uma disfunção local do sistema imunológico; teoria da metaplasia celômica, na qual células pluripotentes sofreriam transformação metaplasmática para tecido endometriótico; teoria da propagação linfovascular, em que células endometriais seriam difundidas por meio das vias linfáticas e vasculares; e a teoria da indução, na qual estímulos induziriam a diferenciação de células indiferenciadas em endometriais ${ }^{(1-3)}$.

Alguns fatores propiciam o desenvolvimento de endometriose como, grupos familiares, uma vez que comorbidades seguem um padrão hereditário poligênico e multifatorial, mutações genéticas e polimorfismos, defeitos anatômicos como obstruções no escoamento do trato reprodutivo e toxinas ambientais ${ }^{(2)}$.

A classificação de endometriose realizada pela American Fertility Society (AFS) é baseada em profundidade da invasão, bilateralidade, envolvimento ovariano, aderências e comprometimento do fundo de saco de Douglas. Escores de 1-15 são classificados como endometriose mínima ou leve, 1640 como moderada e acima de 41 como severa $^{(1-3)}$. Enquanto que, clinicamente, pode ser classificada em: endometriose peritoneal superficial que está sobre o peritônio, endometriose ovariana cística ou endometrioma e endometriose infiltrante profunda que são os implantes com profundidade maior que cinco milímetros, fibrose e hiperplasia muscular abaixo do peritônio ${ }^{(1)}$.

As principais manifestações clínicas são dor pélvica e infertilidade. A dor pélvica pode se manifestar como dismenorreia, dispareunia, disúria, dor defecatória ou não cíclica. Além de limitar significantemente a qualidade de vida das pacientes, tem como consequência problemas econômicos devido à redução na capacidade laboral e ao aumento dos gastos médicos relacionados à doença ${ }^{(4)}$. A infertilidade poder estar relacionada a defeitos na foliculogênese e embriogênese, alterações endometriais que prejudicam a implantação, bem como anormalidades imunológicas ${ }^{(1-2)}$.

Em grande parte dos casos, há um atraso de três anos desde o início dos sintomas devido à dificuldade no diagnóstico, que tem por base a história clínica e exame físico bem detalhados associados a exames de imagem ${ }^{(1)}$. Sinais como dor à mobilização uterina ao toque bimanual e aumento do volume ovariano nos exames complementares são sugestivos de endometriose. No exame físico, nodulações palpáveis no fórnice vaginal posterior ou septo retovaginal, espessamento dos ligamentos uterossacros e lesões violáceas na vagina são sinais sugestivos de endometriose profunda infiltrativa ${ }^{(3)}$. A avaliação da lesão pode ser realizada por ultrassonografia transvaginal ou ressonância magnética, e o diagnóstico final é rea- lizado por análise patológica da biópsia da lesão por meio de laparoscopia ou laparotomia ${ }^{(1)}$.

Entre as complicações mais comuns da endometriose durante a gravidez incluem parto prematuro, sangramentos obstétricos e hemoperitônio espontâneo ${ }^{(5)}$. Atualmente, a endometriose é considerada o fator de risco mais importante para o hemoperitônio espontâneo durante a gravidez $z^{(5-6)}$.

O objetivo deste artigo é apresentar um relato de caso de uma paciente que sofreu uma ruptura espontânea de artéria uterina por foco de endometriose.

\section{Relato de caso}

E.F.S.I, feminina, 27 anos, primigesta, com idade gestacional (IG) ultrassonográfica de 36 semanas, foi admitida no Hospital São Lucas na cidade de Bandeirantes - PR no dia 5 de outubro de 2013 às 06:50 horas. Paciente com antecedentes de hipotireoidismo controlado (TSH: 1,28 e T4L: 1,30) em uso de Levotiroxina 25 mcg e hidronefrose de rim esquerdo. Na admissão, a paciente queixava-se de dor suprapúbica, tipo cólica, de moderada intensidade, com evolução de 4 horas. Ao exame físico de admissão, apresentava-se em regular estado geral, hipocorada $(++/++++)$, desidratada (++/++++), eupneica (FR: $15 \mathrm{mrpm})$, afebril (T: $\left.36,4^{\circ} \mathrm{C}\right)$, anictérica. Pressão arterial (PA) de 120x80 mmHg, frequência cardíaca de $118 \mathrm{bpm}$, saturação de oxigênio em ar ambiente de $98 \%$. Abdome: gravídico, doloroso à palpação em região suprapúbica, com ausência de dinâmica uterina, batimentos cardíacos fetais (BCF) de $138 \mathrm{bpm}$. Foi realizada analgesia diluindo 1 ampola de Buscopan Composto ${ }^{\mathrm{R}} \mathrm{em} 1000 \mathrm{~mL}$ de soro fisiológico $0,9 \%$ via endovenosa 30 gotas / minuto.

A paciente progrediu com perda da consciência e hipotensão (PA: 80x60 mmHg) às 18:30 horas, com aumento dos BCF para $156 \mathrm{bpm}$ e cianose de extremidades. Foi iniciado oxigenioterapia $4 \mathrm{~L} /$ minuto em névoa e infusão de 1.000 mL de Ringer Lactato. Foram realizados a sondagem vesical, o monitoramento cardíaco e a administração de 5.000.000U de penicilina cristalina endovenosa em bolus para profilaxia de infecção neonatal pelo Streptococcus agalactiae pois havia realizado tratamento de infecção urinária no primeiro trimestre de gestação por este agente. Após a conduta, recuperou a consciência, mas manteve-se em regular estado geral, taquicárdica (FC: 105 bpm), hipotensa (PA: 90x65 mmHg), saturação de oxigênio de $96 \%$, afebril (T: $36,2^{\circ} \mathrm{C}$ ), sudoreica, com dor abdominal intensa e queda importante dos BCF (BCF: 119 bpm). Exames laboratoriais: Hemoglobina: 8,8 g/ dl; Hematócrito: 26\%; Leucócitos: 23.000; Plaquetas: 295.000 e Parcial de urina sem alterações.

Às 20:00 horas, devido ao quadro de abdômen agudo e sinais de choque hipovolêmico, a paciente 
foi encaminhada à sala de cirurgia. Foi submetida à cesárea segmentar transversa sob anestesia geral, com retirada do recém nascido em Apgar zero. A dequitação placentária foi realizada sem alterações e ausência macroscópica de hematoma retroplacentário. Na exploração da cavidade, foi encontrado hemoperitônio em grande quantidade oriundo de sangramento de artéria uterina esquerda rota. Após a ligadura do vaso, foi observado que a área apresentava fibrose e foco suspeito de endometriose, sem outros focos na cavidade abdominal. Em seguida, foi realizada histerectomia subtotal e transfusão sanguínea no intraoperatório, devido a atonia uterina com sangramento intenso.

Durante a cirurgia, o produto de histerectomia subtotal foi acondicionado em recipiente próprio, imerso em formalina $10 \%$ e identificado como I e em outro recipiente com formalina e identificado como II, o material perivascular fibrótico. Ambos foram encaminhados, com a devida requisição e descrição do caso, para o serviço de anatomia patológica.

Após estudo anatomopatológico, o laudo emitido por profissional da área demonstrou na análise macroscópica do recipiente I que era produto de histerectomia parcial com endométrio basal, coágulos sanguíneos e pequenos fragmentos placentários na cavidade uterina. $\mathrm{Na}$ análise microscópica deste material observou-se fibras musculares e intensa reação decidual endometrial, em diversos cortes histológicos da camada muscular uterina não foi evidenciado endométrio infiltrado na musculatura. Na microscopia do material do recipiente II, foi laudado como reação decidual perivascular compatível com foco de endometriose.

O presente relato de caso foi submetido e aprovado pelo Comitê de Ética e Pesquisa da Pontifícia Universidade Católica do Paraná sob protocolo CAAE 44699015.8.0000.0020.

\section{Discussão}

A endometriose é uma afecção clínica recorrente que cursa com diversas complicações ginecológicas e obstétricas, sendo a infertilidade uma das complicações mais comuns. Durante o primeiro trimestre, a endometriose está associada com um risco aumentado de perda fetal causada por gravidez ectópica e aborto espontâneo. Enquanto que no terceiro trimestre gestacional, pode ocorrer uma complicação rara, mas com consequências muitas vezes fatais, o hemoperitônio espontâneo, correspondendo a 120 casos relatados até $1980^{(7-9)}$.

Essa patologia é o principal fator de risco para o hemoperitônio espontâneo durante a gestação. O risco está relacionado ao grau de invasão dos focos, podendo ocorrer em qualquer estágio da endometriose. A maioria dos hemoperitônios que ocorrem durante a gestação tem origem na ruptura uterina, todavia vasos ovarianos e uterinos rotos também causam sangramentos $^{(7)}$. Estudos concluíram que em $90 \%$ dos casos o foco hemorrágico era no paramétrio ou na região posterior do útero, também demostraram que em $80 \%$ dos casos a origem do sangramento é venosa, em 16\% arterial e em $4 \%$ não pode ser identificada ${ }^{(5-6)}$.

Entre as prováveis etiologias dos sangramentos por rompimento espontâneo de vasos uterinos estão: o aumento da pressão venosa na circulação útero-ovariana decorrente da pressão intraabdominal aumentada durante a gestação e parto, e a inflamação de $\operatorname{vasos}^{(8-11)}$. As contrações uterinas e o trabalho de parto causariam a avulsão de tecidos localizados sob as aderências associadas a endometriose ${ }^{(7)}$.

Outra possível causa do hemoperitônio deve-se aos focos de endometriose peritoneal que sofrem modificações devido às alterações hormonais durante a gestação. Altas concentrações séricas de progesterona durante o primeiro trimestre levam a fibrose e a redução da pigmentação característica do tecido endometriótico, assim como a diminuição das concentrações ou sensibilidade ao hormônio resultam em regressão do tecido, ativação de enzimas e sangramento ${ }^{(6,9)}$.

A ruptura espontânea de vasos útero-ovarianos tem como manifestações clínicas a dor abdominal súbita, sinais de choque hipovolêmico e diminuição na concentração de hemoglobina, na ausência de sangramento visível ou antecedentes traumáticos ${ }^{(8,}$ 10-11). De acordo com esse quadro semiológico, artigos propuseram como diagnósticos diferenciais: o descolamento prematuro de placenta, a gravidez ectópica abdominal, a ruptura uterina, a apendicite aguda perfurada, a ruptura do fígado ou baço, a torção de cisto ovariano e a obstrução intestinal ${ }^{(8-9)}$. Para fazer o diagnóstico diferencial entre essas etiologias um exame útil é a ultrassonografia ${ }^{(10)}$.

Os exames de imagem utilizados para auxiliar no diagnóstico de hemoperitônio são a ultrassonografia transvaginal e a tomografia computadorizada, que revelam a presença de líquido na cavidade peritoneal. Entretanto, na maioria das vezes, o diagnóstico definitivo só é evidenciado após laparotomia exploradora $^{(6,12)}$. Estudos documentaram que em 117 casos ocorridos até 1987, 61\% foram diagnosticados antes do parto; $21 \%$ durante o período de pós-parto e $18 \%$ no intraparto ${ }^{(8,11)}$.

Estudos observaram que a ruptura das veias útero-ovarianas pode ocorrer entre a $10^{\mathrm{a}}$ semana até o $21^{\circ}$ dia pós-parto, sendo mais frequente no terceiro trimestre ${ }^{(8,10)}$. Em relação a sua taxa de mortalidade houve um índice de $49,3 \%$, sendo que a ocorrência no intraparto atingiu até $76,3 \%$. Pesquisas recentes mostram que a mortalidade materna reduziu a cerca 
de $4 \%$, contudo a taxa de óbito fetal permanece alta, em cerca de $30 \%(5,6,8,11)$. Devido a esse indicador, o hemoperitônio tem indicação cirúrgica, que pode ser realizada através de uma operação cesariana, seguida de investigação da cavidade a fim de encontrar o foco hemorrágico ${ }^{(8,10)}$.

No caso relatado, a causa do hemoperitônio foi a ruptura da artéria uterina esquerda. Dois artigos relataram casos de hemoperitônio espontâneo resultantes de ruptura da artéria uterina, contudo a causa do mesmo não foi a presença do foco de endometriose na artéria. No artigo de Janicki et al (2002) ${ }^{(13)}$, a causa foi a presença de um endometrioma no ovário esquerdo que rompeu a parede da artéria uterina esquerda, a paciente não estava grávida. A paciente do relato foi submetida a uma laparoscopia que posteriormente foi convertida em uma laparotomia. Durante o procedimento recebeu quatro unidades de transfusão sanguínea, tendo permanecido estável após a ligadura da artéria uterina e salpingooforectomia esquerda. Exame histopatológico comprovou a presença de endometrioma no ovário esquerdo ${ }^{(13)}$. No relato de Ellman $(1963)^{(14)}$, a paciente estava grávida de 32 semanas quando apresentou o hemoperitônio. Foi realizada operação cesariana e o prematuro nasceu em boas condições. A paciente recebeu oito unidades de transfusão sanguínea e foi realizada a ligadura do vaso. A análise patológica dos tecidos removidos da paciente não foi realizada, não se podendo afirmar a causa da ruptura $^{(14)}$.

Conclui-se através deste relato de caso que a endometriose durante a gestação pode levar a

complicações que ocorrem especialmente no primeiro e terceiro trimestre gestacionais, como parto prematuro, sangramentos obstétricos e hemoperitônio espontâneo. Sangramentos causados por ruptura de vasos uterinos ou ovariano devem ser considerados, uma vez que podem comprometer a vida materna e fetal.

\section{Referências}

1. Podgaec S. Endometriose - Coleção FEBRASGO. Rio de Janeiro: Elsevier; 2014. 312p.

2. Schorge JO, Halvorson LM, Bradshaw KD, Schaeffer JI, Hoffman BL, Cunningham FG. Endometriosis. In: Schorge JO, Halvorson LM, Bradshaw KD, Schaeffer JI, Hoffman BL, Cunningham FG, editores. Ginecologia de Williams. Porto Alegre: Artmed; 2011. p.225-43.

3. Nácul AP, Spritzer PM. Aspectos atuais do diagnóstico e tratamento da endometriose. Rev Bras Ginecol Obstetr. 2010;32(6):298-307.

4. Barcena de Arellano ML, Mechsner S. The peritoneum -- an important factor for pathogenesis and pain generation in endometriosis. J Mol Med. 2014; 92(6):595-602.

5. Brosens I, Brosens JJ, Fusi L, Al-Sabbagh M, Kuroda K, Benagiano G. Risks of adverse pregnancy outcome in endometriosis. Fertil Steril. 2012; 98(1):30-5.

6. Brosens IA, Fusi L, Brosens JJ. Endometriosis is a risk factor for spontaneous hemoperitoneum during pregnancy. Fertil Steril. 2009; 92(4):1243-5.

7. Kim T-H, Lee H-H. Hemoperitoneum during pregnancy with endometriosis: report of four cases. Iran J Reprod Med. 2010; 8(2):90-3.

8. Andrés-Orós MP, Castro LS, Roy-Ramos V, Vela-Lete A. Rotura espontánea de variz uterina durante la gestación. Ginecol Obstet Méx. 2010;78(2):128-31.

9. Katorza E, Soriano D, Stockheim D, Mashiach R, Zolti M, Seidman DS, et al. Severe intraabdominal bleeding caused by endometriotic lesions during the third trimester of pregnancy. Am J Obstet Gynecol. 2007;197(5):501.e1-4.

10. González Rosales R, Cerón Saldaña MÁ, Ayala Leal I, Cerda López JA. Rotura espontánea de los vasos uterinos durante el embarazo: comunicación de un caso y revisión bibliográfica. Ginecol Obstet Méx. 2008; 76(4):221-3.

11. Passos F, Calhaz-Jorge C, Graça LM. Endometriosis is a possible risk factor for spontaneous hemoperitoneum in the third trimester of pregnancy. Fertil Steril. 2008;89(1):251-2.

12. Lucey BC, Varghese JC, Anderson SW, Soto JA. Spontaneous hemoperitoneum: a bloody mess. Emerg Radiol. 2007;14(2):6575.

13. Janicki TI, David LJ, Skaf R. Massive and acute hemoperitoneum due to rupture of the uterine artery by erosion from an endometriotic lesion. Fertil Steril. 2002;78(4):879-81.

14. Ellman JS. Spontaneous rupture of a branch of the uterine artery in the third trimester of pregnancy. Obstet Gynecol. 1963; 21(1):75-7.

Trabalho recebido: $07 / 06 / 2018$

Trabalho aprovado: $26 / 07 / 2018$ 